\title{
Variantes climáticas e sua relação com as doenças de origem infecciosa: uma revisão integrativa
}

\author{
Climate variables and their relationship whit diseases of infectious origin: An integrative review \\ Variables climáticas y su relación com enfermidades de origen infeccioso: Una revisión integradora
}

Recebido: 18/01/2021 | Revisado: 23/01/2021 | Aceito: 27/01/2021 | Publicado: 04/02/2021

Tatiana Fabíola da Silva Lima

ORCID: https://orcid.org/0000-0001-5966-9727

Centro Universitário Metropolitano da Amazônia, Brasil

E-mail: tatiana.fs.lima2@gmail.com

Gabriel Fazzi Costa

ORCID: https://orcid.org/0000-0002-9672-5676

Universidade do Estado do Pará, Brasil

E-mail: gabrielfazzi@gmail.com

Susiane Martins Silva

ORCID: https://orcid.org/0000-0001-8863-4443

Centro Universitário Metropolitano da Amazônia, Brasil

E-mail: susianemartins@live.com

Gessica da Silva Farias Viana

ORCID: https://orcid.org/0000-0001-8377-0593

Centro Universitário Metropolitano da Amazônia, Brasil

E-mail: gessicadasilva15@hotmail.com

Brenda Tanielle Dutra Barros

ORCID: https://orcid.org/0000-0002-3184-050X

Centro Universitário Metropolitano da Amazônia, Brasil

E-mail: brendatanielle.enf@gmail.com

Luceny Oliveira Mendes

ORCID: https://orcid.org/0000-0003-0505-9493

Centro Universitário Metropolitano da Amazônia, Brasil

E-mail: lumemetais08@hotmail.com

Hayla Cristina de Oliveira Castro

ORCID: https://orcid.org/0000-0002-4041-3067

Centro Universitário Metropolitano da Amazônia, Brasil

E-mail: haylabrinde@hotmail.com

Virgínia Mercês Lara Pessoa Oliveira

ORCID: https://orcid.org/0000-0002-4417-637X

Centro Universitário Metropolitano da Amazônia, Brasil

E-mail: virginia.lara87@gmail.com

Weber Marcos

ORCID: https://orcid.org/0000-0001-7860-2579

Centro Universitário Metropolitano da Amazônia, Brasil

E-mail: weberhmarcos@gmail.com

Maria Karoline Alves Melo

ORCID: https://orcid.org/0000-0001-7428-7188

Secretaria Municipal de Saúde, Brasil

E-mail: mariakarolinea.melo@gmail.com.br

\begin{abstract}
Resumo
Os fatores antropogênicos como deslocamento de populações, aglomerações e o processo acelerado e precário de urbanização em consonância aos fatores climáticos criam condições para aumento rápido de doenças transmissíveis, principalmente as de origem infecciosa. Este estudo corrobora com a ideia de que as variações do clima podem causar efeitos indesejáveis sobre o estado saudável do homem, de formato direto e/ou indireto. O objetivo da presente revisão foi investigar e analisar os estudos existentes na literatura acerca da relação existente entre as variantes climáticas e as doenças de origem infecciosa. Trata-se de uma revisão bibliográfica qualitativa, descritiva, do tipo Revisão Integrativa da Literatura, baseada em investigações científicas divulgadas entre 2015 a 2020. Efetuou-se buscas nas bases de dados: SciELO, LILACS e MEDLINE. A coleta dos estudos foi posta a um instrumento do formulário autenticado por Ursi e Galvão (2015) adaptado. Para análise das informações, foi utilizada a Análise de Conteúdo Temático de Bardin (ACTB). Identificaram-se no total 220 publicações, sendo 203 no MEDLINE, 14 no LILACS e 03 na SciELO. Amostra final foram de 13 produções. O quantitativo de pesquisas brasileiras acerca da temática foi inferior ao esperado, o que é capaz de ser justificado, talvez, pela escolha das palavras-chave. A temperatura, a umidade, a
\end{abstract}


intervenção antropogênica entre outros, alteram ou influenciam de alguma forma na disseminação desta infecção, isto acende advertência para a importância de uma aproximação inter e multidisciplinar, mas partindo uma perspectiva ética sustentável e justa que permita a convivência digna e equilibrada com todos os seres vivos.

Palavras-chave: Mudanças climáticas; Doenças infecciosas; Epidemiologia.

\begin{abstract}
The anthropogenic factors such as displacement of populations, agglomerations and the accelerated and precarious process of urbanization in line with climatic factors create conditions for the rapid increase in communicable diseases, especially those of infectious origin. This study corroborates the idea that weather variations can cause undesirable effectsabout the healthy state of man, in direct and / or indirect format. The purpose of this review was to investigate and analyze the studies in the literature about the relationship betweenclimatic variantsand diseases of infectious origin. Its about a review qualitative, descriptive bibliography, such as Integrative Literature Review, based on scientific investigations disclosed between 2015 to 2020. Andfetched searchesin the databases: SciELO, LILACS and MEDLINE. Collection of studieswas put to a form instrument authenticated by Ursi and Galvão (2015) Adapted. For analysis of information, Bardin Thematic Content Analysis (ACTB) was used. A total of 220 publications were identified, 203 in MEDLINE, 14 in LILACS and 03 in SciELO. Final sample was 13 productions.O quantitative Brazilian research on the theme was lower than expected, which is able toperhaps justified by the choice of keywords. Temperature, humidity, anthropogenic intervention, among others, alter or influence in some way the spread of this infection, this turns onwarning for the importance of a approximation inter and multidisciplinarybut leaving a sustainable and fair ethical perspective that allows dignified and balanced coexistence with all living beings.
\end{abstract}

Keywords: Climate changes; Infectious diseases; Epidemiology.

\title{
Resumen
}

Los factores antropogénicos como el desplazamiento de poblaciones, las aglomeraciones y el acelerado y precario proceso de urbanización en consonancia con los factores climáticos crean las condiciones para el rápido aumento de las enfermedades transmisibles, especialmente las de origen infeccioso. Este estudio corrobora la idea de que las variaciones climáticas pueden causar efectos indeseablessobre el estado de salud del hombre, en formato directo y/o indirecto. El propósito de esta revisión fue investigar y analizar los estudios en la literatura sobre la relación entrevariantes climáticasy enfermedades de origen infeccioso. Se trata de una revisión Bibliografía descriptiva cualitativa, como Integrative Literature Review, basada en investigaciones científicas divulgado entre 2015 y 2020. Ybúsquedas obtenidasen las bases de datos: SciELO, LILACS y MEDLINE. Colección de estudiosfue puesto a un instrumento de forma autenticado por Ursi y Adaptado Galvão (2015). Para análisis de información, Se utilizó el análisis de contenido temático Bardin (ACTB). Se identificaron un total de 220 publicaciones, 203 en MEDLINE, 14 en LILACS y 03 en SciELO. La muestra final fue de 13 producciones.O cuantitativo La investigación brasileña sobre el tema fue menor de lo esperado, lo que es capaz dequizás justificado por la elección de palabras clave. La temperatura, la humedad, la intervención antropogénica, entre otros, alteran o influyen de alguna manera en la propagación de esta infección, esta se enciendeadvertencia por la importancia de un aproximación inter y multidisciplinarpero dejando una perspectiva ética sostenible y justa que permita la convivencia digna y equilibrada con todos los seres vivos.

Palabras clave: Cambios climáticos; Enfermedades infecciosas; Epidemiología.

\section{Introdução}

Compreende-se a diversidade climática como um fenômeno complexo e dinâmico que envolve aspectos sociais, físicos e ambientais. O desflorestamento, em combinação com as modificações climáticas relativas às mudanças de quantidade de calor presente na atmosfera, umidade do ar e variação pluviométrica contribuem com o risco de proliferação e disseminação de diversas doenças. As alterações ambientais têm enorme impacto no surgimento e ressurgimento de doenças, sobretudo em países com alta biodiversidade e que enfrentam sérias questões ambientais, sociais e econômicas, como ocorre com o Brasil (Abreu et al., 2020).

Este estudo corrobora com a ideia de que as variedades do clima têm relação diretamente proporcional aos impactos sobre os seres humanos, principalmente no que tange a sua saúde, e que estes impactos atuam sobre um formato direto e/ou indireto. Lobão e Rodrigues (2019) explicam que, acontece em formato direto, via aumento de calor ou eventos extremos, ou indiretamente, por modificações ambientais como por exemplo a alteração junto a modificação de ecossistemas e de períodos ou etapas cíclicas biogeoquímicas. As mudanças ecossistêmicas podem trazer problemas como contingência de comorbidades infecciosas e aumento de vetores. 
A escolha do tema também prende-se a o fato de que o denso deslocamento de populações, as aglomerações e o processo acelerado e precário de urbanização também criam condições para propagação de patologias transmissíveis. Situações como estas também contribuem para o surgimento diversas doenças, as quais têm envolvido números crescentes de vítimas com sintomas cada vez mais graves (Duarte et al., 2019). Desta forma, questiona-se: Qual a relação das variações climáticas com as doenças de origem infecciosa?

Esta pesquisa justifica-se pelas suas possíveis contribuições, em âmbito acadêmico, social e ambiental, ao tratar dos impactos ambientais ocasionados a partir das mudanças do clima, que influenciam e interferem na saúde e ecossistema.

Neste contexto, o objetivo da presente revisão integrativa da literatura foi investigar e analisar os estudos acerca da relação existente entre as variantes climáticas e as doenças de origem infecciosa, além de investigar os principais impactos da variação climática sobre o bem estar humano.

\section{Metodologia}

Este estudo pautou-se em uma pesquisa de abordagem qualitativa, descritiva, do tipo Revisão Integrativa da Literatura (RIL), baseada em estudos científicos publicados de 2015 a 2020. A revisão integrativa faz parte da categoria das revisões sistemáticas, esta permite demonstrar de forma mais ampla e abrangente a literatura já divulgada, tendo como base distintos conceitos advindos de saberes científicos ou empíricos, promovendo uma vista mais ampla sobre um determinado estudo (Barros et al., 2021). Já as metodologias de formato qualitativo são aquelas primordiais no que tange a interpretação do autor ou autores da pesquisa com suas concepções a partir do fenômeno em pauta (Pereira et al., 2018).

Para a investigação e coleta dos artigos na literatura, realizou-se buscas nas bases de dados: SciELO, LILACS e MEDLINE. Foram utilizados, para busca dos artigos, as palavras-chave "Mudanças climáticas", "Doenças infecciosas" e "Epidemiologia".

A partir desse conjunto de palavras-chave e para a busca dos artigos, através dos filtros das próprias bases de dados, estabeleceram-se os seguintes critérios de inclusão: artigos disponíveis na integra, em português, inglês e espanhol, no período de 2015 a 2020 com acesso gratuito e que tivessem afinidade com a temática. Foram definidos como critérios de exclusão: livros digitais, resumos, monografias, dissertações, teses, artigos duplicados e fora do período definido para o estudo e que não se adequassem aos objetivos da pesquisa.

As referências obtidas foram importadas para o Software Mendeley, que foi utilizado para excluir as repetições entre as bases de dados. Após isto, as referências foram transferidas para o Programa Excel-Windows 10, para a dupla conferência da existência de duplicações.

$\mathrm{Na}$ coleta dos dados utilizou-se um instrumento do formulário validado por Ursi \& Galvão (2015) adaptado. Neste instrumento, após leitura minuciosa, os dados extraídos foram transcritos, possibilitando o detalhamento dos estudos que, como exemplo, podem ser organizados por tabelas em ordem numérica crescente, por exemplo, em comum acordo com o ano de publicação e o título da pesquisa (Lima et al., 2020; Teixeira et al., 2013).

Para análise dos dados, foi utilizada a Análise de Conteúdo Temático de Bardin (ACTB). As diferentes fases da análise de conteúdo organizam-se em torno de três polos cronológicos: A pré-análise, exploração do material e o tratamento dos resultados (Bardin, 2016).

A primeira etapa da avaliação das referências constou da leitura dos títulos dos artigos, a segunda da leitura dos resumos, e a terceira da leitura crítica e avaliação das pesquisas eleitas. Em todas as etapas, a leitura e a análise dos artigos tiveram a dupla conferência para evitar vieses de seleção. Na primeira e na segunda etapas excluíram-se os estudos que não tivessem como desfecho ou agravos uma ou mais variáveis climáticas e que não explicitassem quais mudanças interferiam na 
frequência e na gravidade de patologias infecciosas ou que dessem enfoque apenas a uma variável climática e uma única doença infecciosa.

$\mathrm{Na}$ terceira etapa, os artigos foram lidos na íntegra e, para se verificar a pertinência de sua inclusão, utilizou-se as informações: língua (português, inglês e espanhol), resultados e discussões, país da pesquisa, relação com as adversidades ambientais e climáticas que não fossem estudos referentes a zoologia, botânica, clínica médica, bacteriologia e o desfecho.

\section{Resultados e Discussão}

Identificaram-se no total 220 publicações, sendo 203 no MEDLINE, 14 no LILACS e 03 na SciELO. Destes, foram encontrados 5 estudos duplicados, permanecendo 215 estudos. Após aplicação dos critérios de inclusão, a amostra foi de 107 produções. Após avaliação crítica do título, resumo, desfecho final e leitura da sua relação com os objetivos do estudo, a amostra final foram de 13 produções, sendo 11 na MEDLINE, 02 na SCIELO e, na LILACS, nenhum artigo contemplava o objetivo da pesquisa. Os resultados apresentados acima foram dispostos ao Fluxograma 1 PRISMA (Moher et al., 2009).

Fluxograma 1. Fluxograma PRISMA com as etapas de inclusão dos artigos.
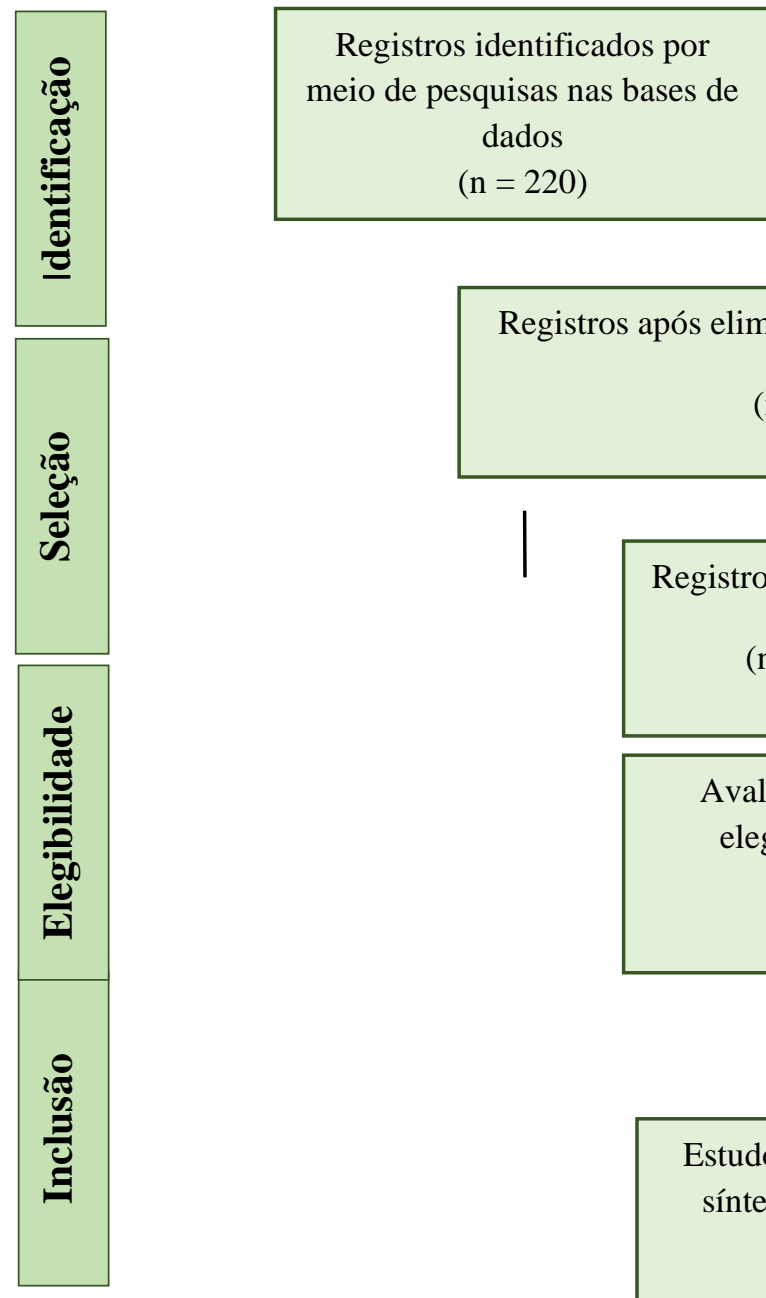

Registros identificados por meio de pesquisas em outras fontes de dados $(n=5)$

Registros após eliminar os estudos duplicados

$(\mathrm{n}=215)$

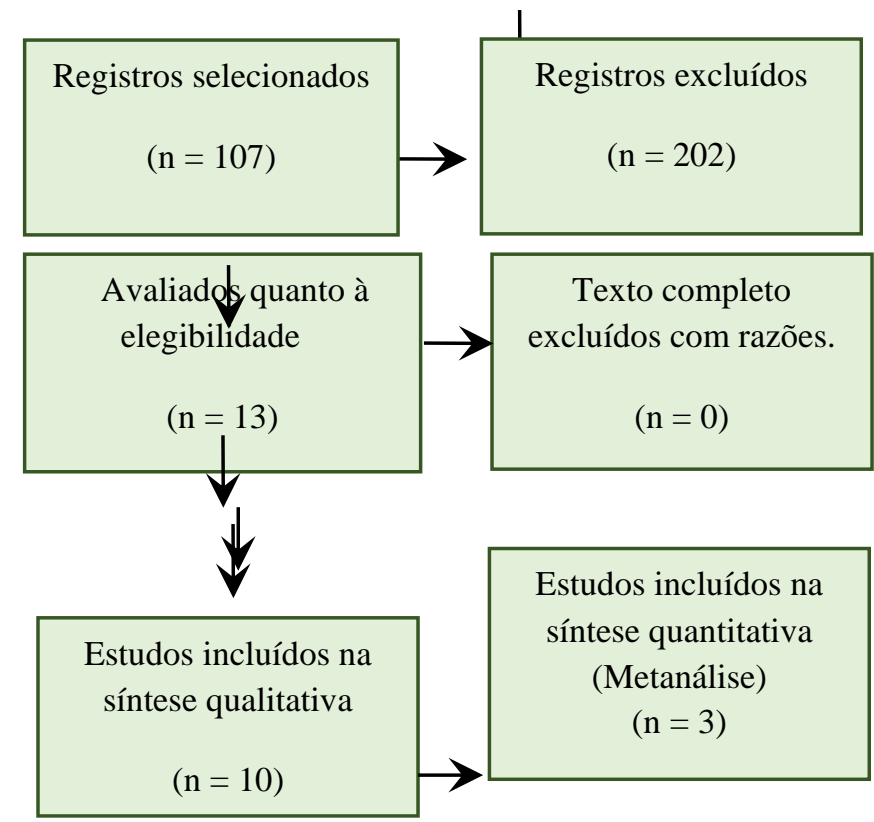

Fonte: Adaptado de Moher, D., et al. (2009). 
No Fluxograma 1, foi possível observar, detalhadamente, o quantitativo de artigos encontrados e que a maioria dos estudos selecionados versam sobre as pesquisas de síntese qualitativa e a menoria, incluídas na síntese quantitativa do tipo metanálise.

As produções foram agrupadas e dispostas através do instrumento adaptado de Ursi (2015). Optou-se por selecionar os itens: Autor/ano/Periódico; Título; Objetivo; Resultados; Categoria/Referência ( N $^{\circ}$ ), (Quadro 1).

Quadro 1. Produções no período de 2015 a 2020.

\begin{tabular}{|c|c|c|c|c|}
\hline $\begin{array}{l}\text { Autor/Ano/ } \\
\text { Periódico }\end{array}$ & Título & Objetivo & Resultados & $\begin{array}{c}\text { Categoria/ } \\
\text { Referência }\left(\mathbf{N}^{o}\right)\end{array}$ \\
\hline $\begin{array}{l}\text { SABIN, N. S., et al. } \\
\text { (2020). } \\
\text { Journal Of } \\
\text { Physiological } \\
\text { Anthropology }\end{array}$ & $\begin{array}{l}\text { Implications of } \\
\text { human activities } \\
\text { for (re)emerging } \\
\text { infectious } \\
\text { diseases, } \\
\text { including } \\
\text { COVID-19 }\end{array}$ & $\begin{array}{l}\text { Descrever fatores } \\
\text { relacionados às atividades } \\
\text { humanas e como elas podem } \\
\text { desempenhar um papel na } \\
\text { transmissão e disseminação } \\
\text { de doenças infecciosas. } \\
\text { Descrever e discutir os } \\
\text { principais fatores que estão } \\
\text { facilitando a propagação da } \\
\text { nova pandemia conhecida } \\
\text { como COVID-19 em todo o } \\
\text { mundo. }\end{array}$ & $\begin{array}{l}\text { Atividades humanas que influenciam na } \\
\text { transmissão e disseminação de doenças } \\
\text { infecciosas: Globalização; Mudanças no } \\
\text { ambiente; mudanças ecológicas; } \\
\text { Interface homem-animal selvagem; } \\
\text { Resistência a antimicrobiana; } \\
\text { Suscetibilidade ao hospedeiro; } \\
\text { Mudanças de comportamento. }\end{array}$ & $\begin{array}{c}\text { Mudanças } \\
\text { climáticas e } \\
\text { atividades } \\
\text { humanas como } \\
\text { determinantes } \\
\text { significativas nas } \\
\text { doenças } \\
\text { infecciosas. } \\
\mathbf{N}^{\mathbf{0}} \mathbf{1}\end{array}$ \\
\hline $\begin{array}{l}\text { CASADEVALL, } \\
\text { A., et al. (2020). } \\
\text { Journal Of Clinical } \\
\text { Investigation }\end{array}$ & $\begin{array}{l}\text { Climated change } \\
\text { brings the specter } \\
\text { of new infectious } \\
\text { diseases. }\end{array}$ & $\begin{array}{l}\text { Apresentar o ponto de vista: } \\
\text { a forte possibilidade de que } \\
\text { novas doenças infecciosas } \\
\text { desconhecidas surjam de } \\
\text { climas mais quentes à } \\
\text { medida que os patógenos se } \\
\text { adaptam a temperaturas } \\
\text { globais mais altas que } \\
\text { podem derrotar nossa } \\
\text { barreira } \\
\text { endotérmica. }\end{array}$ & $\begin{array}{l}\text { Medidas tomadas para aumentar a } \\
\text { preparação para enfrentar novas } \\
\text { doenças infecciosas: Aumentar a } \\
\text { vigilância para novas doenças; } \\
\text { promover a pesquisa sobre as interações } \\
\text { patógeno-hospedeiro; promover o } \\
\text { desenvolvimento contínuo de } \\
\text { terapêuticas } \\
\text { desenvolver matrizes para identificar } \\
\text { novas ameaças potenciais prováveis. }\end{array}$ & $\begin{array}{l}\text { Doenças } \\
\text { infecciosas e sua } \\
\text { relação com as } \\
\text { mudanças de } \\
\text { temperatura } \\
\mathbf{N}^{\mathbf{0}} \mathbf{2}\end{array}$ \\
\hline $\begin{array}{l}\text { OMAZIC, A., et al. } \\
\text { (2019). } \\
\text { Acta Veterinaria } \\
\text { Scandinavica }\end{array}$ & $\begin{array}{l}\text { Identifying } \\
\text { climate-sensitive } \\
\text { infectious } \\
\text { diseases in } \\
\text { animals and } \\
\text { humans in } \\
\text { Northern regions }\end{array}$ & $\begin{array}{l}\text { Identificar doenças } \\
\text { infecciosas sensíveis ao } \\
\text { clima (CSIs) de relevância } \\
\text { para humanos e / ou animais } \\
\text { que vivem nas regiões do } \\
\text { Norte. }\end{array}$ & $\begin{array}{l}\text { De } 1275 \text { foram obtidos os resultados: } \\
\text { doenças transmitidas por vetores de } \\
\text { artrópodes são reconhecidas como } \\
\text { tendo potencial para expandir sua } \\
\text { distribuição para as latitudes do norte; a } \\
\text { encefalite e borreliose transmitidas por } \\
\text { carrapatos, febre catarral ovina e a } \\
\text { infecção parasitária fasciolose podem } \\
\text { ser classificadas como sensíveis ao } \\
\text { clima. }\end{array}$ & $\begin{array}{c}\text { A influência do } \\
\text { clima sobre os } \\
\text { patógenos e } \\
\text { hospedeiros/ } \\
\text { vetores } \\
\mathbf{N}^{\mathbf{0}} \mathbf{3}\end{array}$ \\
\hline $\begin{array}{l}\text { KHAN, M. D., et } \\
\text { al., (2019). } \\
\text { International Journal } \\
\text { Of Environmental } \\
\text { Research And Public } \\
\text { Health }\end{array}$ & $\begin{array}{l}\text { Aggravation of } \\
\text { Human Diseases } \\
\text { and Climate } \\
\text { Change Nexus }\end{array}$ & $\begin{array}{lr}\text { Examinar e } & \text { discutir } \\
\text { principalmente } & \text { impacto } \\
\text { das mudanças } & \text { climáticas } \\
\text { com base em } & \text { evidências } \\
\text { científicas na } & \text { literatura } \\
\text { publicada } & \end{array}$ & $\begin{array}{l}\text { Com base em estudos realizados entre } \\
\text { 1990-2018, três aspectos que se } \\
\text { assemelham ao impacto da mudança } \\
\text { climática sobre as doenças: a) } \\
\text { emergência e reemergência de doenças } \\
\text { transmitidas por vetores, b) impacto de } \\
\text { eventos climáticos extremos, e c) } \\
\text { elevação social com educação e } \\
\text { adaptação. }\end{array}$ & $\begin{array}{c}\text { A influência do } \\
\text { clima sobre os } \\
\text { patógenos e } \\
\text { hospedeiros/ } \\
\text { vetores } \\
\mathbf{N}^{0} \mathbf{4}\end{array}$ \\
\hline $\begin{array}{c}\text { SOUSA, T. C. M. } \\
\text { de., et al. (2018). } \\
\text { Revista } \\
\text { Panamericana de }\end{array}$ & $\begin{array}{l}\text { Doenças sensíveis } \\
\text { ao clima no Brasil } \\
\text { e no mundo: } \\
\text { revisão } \\
\text { sistemática }\end{array}$ & $\begin{array}{l}\text { Fazer um levantamento da } \\
\text { literatura existente acerca } \\
\text { das doenças sensíveis ao } \\
\text { clima (DSC) e dos impactos } \\
\text { das alterações climáticas }\end{array}$ & $\begin{array}{l}\text { Foram selecionadas } 106 \text { publicações. } \\
\text { Doenças mais estudadas: dengue, } \\
\text { malária, doenças respiratórias e } \\
\text { cardiovasculares. Variáveis climáticas: } \\
\text { temperatura, precipitação. Relação entre }\end{array}$ & $\begin{array}{l}\text { A influência do } \\
\text { clima sobre os } \\
\text { patógenos e } \\
\text { hospedeiros/ } \\
\text { vetores }\end{array}$ \\
\hline
\end{tabular}




\begin{tabular}{|c|c|c|c|c|}
\hline Salud. Pública & & sobre a saúde. & $\begin{array}{l}\text { incidência de doenças, cardiovasculares } \\
\text { e respiratórias, dengue, malária e } \\
\text { arboviroses às condições climáticas em } \\
\text { diferentes regiões do mundo. O número } \\
\text { maior de estudos foram realizados por } \\
\text { autores de países desenvolvidos. Locais } \\
\text { mais estudados: China, EUA, Austrália } \\
\text { e Brasil. }\end{array}$ & $N^{0} 5$ \\
\hline $\begin{array}{l}\text { NAVA, A., et al. } \\
\text { (2017). } \\
\text { Ilar Journal }\end{array}$ & $\begin{array}{l}\text { The Impact of } \\
\text { Global } \\
\text { Environmental } \\
\text { Changes on } \\
\text { Infectious } \\
\text { Disease } \\
\text { Emergence with a } \\
\text { Focus on Risks } \\
\text { for Brazil }\end{array}$ & $\begin{array}{l}\text { Resumir os achados mais } \\
\text { importantes com maior } \\
\text { atenção para o Brasil e as } \\
\text { doenças de atual importância } \\
\text { para a saúde pública }\end{array}$ & $\begin{array}{l}\text { A literatura revelou uma relação entre } \\
\text { surtos de doenças infecciosas e eventos } \\
\text { de mudanças climáticas (El Niño, La } \\
\text { niña, ondas de calor, secas, inundações, } \\
\text { aumento de temperatura, maior } \\
\text { precipitação) ou mudanças ambientais } \\
\text { (fragmentação de habitat, } \\
\text { desmatamento, urbanização, consumo } \\
\text { de carne de caça e outros). }\end{array}$ & $\begin{array}{c}\text { Mudanças } \\
\text { climáticas e } \\
\text { atividades } \\
\text { humanas como } \\
\text { determinantes } \\
\text { significativas nas } \\
\text { doenças } \\
\text { infecciosas. } \\
\mathbf{N}^{\mathbf{0}} \mathbf{6}\end{array}$ \\
\hline $\begin{array}{l}\text { METCALF, C. J. } \\
\text { E., et al. (2017). } \\
\text { Proceedings Of The } \\
\text { Royal Society B: } \\
\text { Biological Sciences }\end{array}$ & $\begin{array}{l}\text { Identifying } \\
\text { climate drivers of } \\
\text { infectious disease } \\
\text { dynamics: recent } \\
\text { advances and } \\
\text { challenges ahead }\end{array}$ & $\begin{array}{l}\text { Detalhar os mecanismos } \\
\text { pelos quais os fatores } \\
\text { climáticos podem moldar a } \\
\text { incidência de doenças } \\
\text { infecciosas, efeitos direto no } \\
\text { vetor a efeitos indiretos na } \\
\text { suscetibilidade humana. }\end{array}$ & $\begin{array}{l}\text { Relações entre condições climáticas e } \\
\text { doenças infecciosas podem ser } \\
\text { observadas em uma faixa de escalas } \\
\text { espaciais e temporais; Doenças } \\
\text { infecciosas podem ser transmitidas } \\
\text { diretamente através de partículas } \\
\text { transmitidas pelo ar ou fômites ou } \\
\text { transmitidas indiretamente por } \\
\text { alimentos, água ou vetor e podem } \\
\text { potencialmente envolver espécies de } \\
\text { reservatórios; As consequências da } \\
\text { variação climática podem variar desde a } \\
\text { transmissão até a mudança da } \\
\text { magnitude da transmissão. }\end{array}$ & $\begin{array}{l}\text { A influência do } \\
\text { clima sobre os } \\
\text { patógenos e } \\
\text { hospedeiros/ } \\
\text { vetores } \\
\mathbf{N}^{\mathbf{7}} \mathbf{7}\end{array}$ \\
\hline $\begin{array}{l}\text { FISMAN, D. N.; } \\
\text { TUITE, A. R.; } \\
\text { BROWN, K. A. } \\
\text { (2016). } \\
\text { Proceedings Of The } \\
\text { National Academy } \\
\text { Of Sciences }\end{array}$ & $\begin{array}{l}\text { Impact of el niño } \\
\text { southern } \\
\text { oscillation on } \\
\text { infectious disease } \\
\text { hospitalization } \\
\text { risk in the United } \\
\text { State }\end{array}$ & $\begin{array}{l}\text { Avaliar as associações entre } \\
\text { ENOS e risco de } \\
\text { hospitalização por doenças } \\
\text { infecciosas em várias regiões } \\
\text { dos EUA, e avaliar a } \\
\text { heterogeneidade entre } \\
\text { regiões e, identificar as } \\
\text { diferenças no efeito de } \\
\text { ENOS entre as regiões } \\
\text { ocidentais conectadas dos } \\
\text { EUA. }\end{array}$ & $\begin{array}{l}\text { O índice ENSO foi mais associado com } \\
\text { doenças transmitidas por vetores na } \\
\text { região oeste; o aumento nas doenças } \\
\text { transmitidas por vetores foi atribuível } \\
\text { ao aumento do risco de rickettsioses e } \\
\text { doenças infecciosas transmitidas por } \\
\text { carrapatos. Em contraste, ENSO foi } \\
\text { associado a mais doença entérica em } \\
\text { regiões não ocidentais }\end{array}$ & $\begin{array}{l}\text { Doenças } \\
\text { infecciosas e sua } \\
\text { relação com as } \\
\text { mudanças de } \\
\text { temperatura. } \\
\mathbf{N}^{\mathbf{0}} \mathbf{8}\end{array}$ \\
\hline $\begin{array}{l}\text { WU, X., et al. } \\
\text { (2016). } \\
\text { Environment } \\
\text { International }\end{array}$ & $\begin{array}{l}\text { Impact of climate } \\
\text { change on human } \\
\text { infectious } \\
\text { diseases: } \\
\text { Empirical } \\
\text { evidence and } \\
\text { human adaptation }\end{array}$ & $\begin{array}{l}\text { Examinar as evidências } \\
\text { científicas sobre o impacto } \\
\text { das mudanças climáticas nas } \\
\text { doenças infecciosas } \\
\text { humanas. Ele identifica o } \\
\text { progresso da pesquisa e as } \\
\text { lacunas sobre como a } \\
\text { sociedade humana pode } \\
\text { responder, se adaptar e se } \\
\text { preparar para as mudanças } \\
\text { relacionadas. }\end{array}$ & $\begin{array}{l}\text { Publicações relacionadas entre } 1990 \text { e } \\
2015 \text {, refletem } 3 \text { aspectos: os } \\
\text { componentes das doenças infecciosas; } \\
\text { as variáveis climáticas; doenças } \\
\text { infecciosas. Medidas de adaptação às } \\
\text { mudanças climáticas: 1) ir além das } \\
\text { observações empíricas; 2) melhorar a } \\
\text { previsão do processo espaço-temporal } \\
\text { das mudanças climáticas e as associadas } \\
\text { doenças infecciosas; 3) estabelecer uma } \\
\text { melhor compreensão dos padrões de } \\
\text { mudança climática e dos efeitos de } \\
\text { saúde e recursos para promover estilos } \\
\text { de vida saudáveis e consciência pública. }\end{array}$ & $\begin{array}{l}\text { A influência do } \\
\text { clima sobre os } \\
\text { patógenos e } \\
\text { hospedeiros/ } \\
\text { vetores } \\
\mathbf{N}^{\mathbf{9}} \mathbf{9}\end{array}$ \\
\hline $\begin{array}{l}\text { LIMA-CAMARA, } \\
\text { T. N. (2016). } \\
\text { Revista de Saúde } \\
\text { Pública }\end{array}$ & $\begin{array}{l}\text { Arboviroses } \\
\text { emergentes e } \\
\text { novos desafios } \\
\text { para a saúde } \\
\text { pública no Brasil }\end{array}$ & $\begin{array}{l}\text { Discutir a recente entrada } \\
\text { dos arbovírus Chikungunya } \\
\text { (CHIKV), Febre do Oeste do } \\
\text { Nilo (WNV) e Zika (ZIKV) } \\
\text { no Brasil, com enfoque nos } \\
\text { desafios para a Saúde } \\
\text { Pública do País. }\end{array}$ & $\begin{array}{l}\text { No final de } 2014 \text {, o Brasil registrou a } \\
\text { circulação do Chikungunya; A primeira } \\
\text { evidência sorológica da Febre do Oeste } \\
\text { do Nilo ocorreu em 2009. Em 2015, } \\
\text { surgiram os primeiros casos de Zika; } \\
\text { Desafios para a saúde pública: controle } \\
\text { do vetor de dengue, Chikungunya e }\end{array}$ & $\begin{array}{c}\text { Mudanças } \\
\text { climáticas e } \\
\text { atividades } \\
\text { humanas como } \\
\text { determinantes } \\
\text { significativas nas } \\
\text { doenças }\end{array}$ \\
\hline
\end{tabular}




\begin{tabular}{|c|c|c|c|c|}
\hline & & & $\begin{array}{l}\text { Zika; crescimento desordenado das } \\
\text { cidades, poluição de rios e formação de } \\
\text { valas; o diagnóstico de novas } \\
\text { arboviroses. }\end{array}$ & $\begin{array}{c}\text { infecciosas } \\
\mathbf{N}^{\mathbf{0}} \mathbf{1 0}\end{array}$ \\
\hline $\begin{array}{l}\text { GISLASON, M. } \\
\text { K., et al. (2015). } \\
\text { Virulence }\end{array}$ & $\begin{array}{l}\text { Climate change, } \\
\text { health and } \\
\text { infectious disease }\end{array}$ & $\begin{array}{l}\text { Enquadrar os danos à saúde } \\
\text { causados pelo clima como } \\
\text { fenômenos socioecológicos e } \\
\text { argumentando que a saúde } \\
\text { pública deve não apenas } \\
\text { controlar e conter, mas } \\
\text { também buscar prevenir o } \\
\text { surgimento de doenças } \\
\text { causadas pelo clima. }\end{array}$ & $\begin{array}{l}\text { Riscos de mudanças climáticas para a } \\
\text { saúde: Primárias: Consequências } \\
\text { biológicas de ondas de calor, eventos } \\
\text { climáticos extremos e aumento de } \\
\text { temperatura; Secundário: Riscos } \\
\text { mediados por mudanças em processos e } \\
\text { sistemas de base biofísica e ecológica, } \\
\text { produção de alimentos, fluxos de água, } \\
\text { vetores de doenças infecciosas e } \\
\text { ecologia de hospedeiro intermediário; } \\
\text { Terciário: Consequências de tensão e } \\
\text { conflito devido aos declínios } \\
\text { relacionados com as mudanças } \\
\text { climáticas nos recursos básicos. }\end{array}$ & $\begin{array}{c}\text { Mudanças } \\
\text { climáticas e } \\
\text { atividades } \\
\text { humanas como } \\
\text { determinantes } \\
\text { significativas nas } \\
\text { doenças } \\
\text { infecciosas. } \\
\mathbf{N}^{\mathbf{0}} \mathbf{1 1}\end{array}$ \\
\hline \begin{tabular}{l} 
CONFALONIERI, \\
U. E. C.; \\
MENEZES, J. A. \& \\
SOUZA, C. M. \\
de., et al. (2015). \\
\multicolumn{2}{c}{ Virulence }
\end{tabular} & $\begin{array}{l}\text { Climate change } \\
\text { and adaptation of } \\
\text { the health sector: } \\
\text { The case of } \\
\text { infectious } \\
\text { diseases }\end{array}$ & $\begin{array}{l}\text { Revisar a questão de como } \\
\text { os sistemas de saúde e os } \\
\text { programas de controle de } \\
\text { doenças infecciosas devem } \\
\text { mudar suas práticas a fim de } \\
\text { lidar com os crescentes } \\
\text { desafios colocados pelas } \\
\text { mudanças climáticas globais }\end{array}$ & $\begin{array}{l}\text { A proteção à saúde da população } \\
\text { humana das mudanças na epidemiologia } \\
\text { de doenças infecciosas ocorridas em } \\
\text { consequência das mudanças climáticas } \\
\text { envolve ações nos sistemas de saúde e } \\
\text { em outros setores. Na saúde são } \\
\text { necessárias estratégias como vigilância } \\
\text { epidemiológica e entomológica } \\
\text { aprimorada e direcionada e o } \\
\text { desenvolvimento de sistemas de alerta } \\
\text { precoces de epidemias informados pelos } \\
\text { cenários climáticos. }\end{array}$ & $\begin{array}{l}\text { A influência do } \\
\text { clima sobre os } \\
\text { patógenos e } \\
\text { hospedeiros/ } \\
\text { vetores } \\
\mathbf{N}^{\mathbf{0}} \mathbf{1 2}\end{array}$ \\
\hline $\begin{array}{l}\text { WANG, Y., et al. } \\
\text { (2015). } \\
\text { International Journal } \\
\text { Of Environmental } \\
\text { Research And Public } \\
\quad \text { Health }\end{array}$ & $\begin{array}{l}\text { A Method for } \\
\text { Screening } \\
\text { Climate Change- } \\
\text { Sensitive } \\
\text { Infectious } \\
\text { Diseases }\end{array}$ & $\begin{array}{l}\text { Propor um método } \\
\text { estatisticamente significativo } \\
\text { (relative risk }-\mathrm{RR} \text { ) e } \\
\text { abrangente para identificar } \\
\text { quais doenças infecciosas } \\
\text { são sensíveis às mudanças } \\
\text { climáticas. }\end{array}$ & $\begin{array}{l}\text { Resultados consideraram três variáveis } \\
\text { climáticas: temperatura média mensal, } \\
\text { precipitação acumulada mensal e } \\
\text { umidade absoluta média mensal. Os } \\
\text { resultados sugeriram que seis doenças } \\
\text { infecciosas foram afetadas pela } \\
\text { mudança de temperatura em mais de } \\
50 \% \text { dos condados e cidades efetivas na } \\
\text { Província de Anhui de } 2004 \text { a } 2010 \text {; são } \\
\text { eles: HFM, malária, gripe, febre tifóide, } \\
\text { meningite e esquistossomose. }\end{array}$ & $\begin{array}{l}\text { A influência do } \\
\text { clima sobre os } \\
\text { patógenos e } \\
\text { hospedeiros/ } \\
\text { vetores } \\
\mathbf{N}^{\mathbf{0}} \mathbf{1 3}\end{array}$ \\
\hline
\end{tabular}

Fonte: Adaptado de Ursi, E. S. \& Galvão, C. M. (2015).

Na Tabela 1 observa-se os artigos dispostos em ordem cronológica decrescente, com as respectivas referências numeradas $\left(\mathrm{N}^{\circ}\right)$ em ordem crescente, onde foram selecionados, analisados e distribuídos conforme seus conteúdos em três categorias: 1) Mudanças climáticas e atividades humanas como determinantes significativas nas doenças infecciosas, 2) Doenças infecciosas e sua relação com as variações de temperatura e 3) A influência do clima sobre os patógenos e hospedeiros/vetores.

Na primeira categoria, foram adicionados os 04 artigos que perpassam pela temática, compreendendo as referências: (1), (6), (10) e (11). Ainda na primeira categoria, 02 artigos possuem enfoque no desflorestamento como um determinante fator antropogênico das variantes climáticas e na transmissão e disseminação de patologias infecciosas, são eles: (1) e (10). Mais 02 estudos enquadram-se na primeira categoria e vêm abordando a interferência e modificação dos ecossistemas e a urbanização como impulsionadores de uma ampla gama de doenças infecciosas potencialmente fatais, são: (6) e (11).

Destaca-se a referência: (11), onde afirma-se que paralelamente, a elevação da temperatura global também se mostra como um importante fator no processo de transmissibilidade de patógenos ao homem. A elevação da temperatura global afeta 
os mosquitos vetores reduzindo o ciclo de desenvolvimento de larvas e aumentando rapidamente a população de vetores adultos.

Destaca-se também outra referência: (10) que mostra a atividade humana como desmatamento, expansão agrícola, invasão de habitat, caça e comércio como mecanismos pelos quais humanos e da vida selvagem entram em novas interações que podem ampliar as oportunidades de transmissão, como exemplo, a Covid-19.

$\mathrm{Na}$ segunda categoria, 02 estudos foram encontrados, compreendendo as referências: (2) e (8). Com foco no aquecimento global e as diversas espécies de fungos, vírus e bactérias que se adaptam a temperaturas altas e se tornam um potencialmente patogênico para os humanos, o artigo de referência (2) contempla esta temática. Também tem-se a referência (8), estudo que retrata a ligação direta entre o ambiente físico e o risco de infecção, que são mais evidentes em países de baixa renda, com escassez de saneamento básico e deficiência na saúde coletiva pública.

Na terceira categoria há 07 estudos atrelados a temática: (3), (4), (5), (7), (9), (12) e (13). que abordam a forte dependência das patologias propagadas por vetores com as condições climáticas. Os artigos (3), (4), (9) inferem que as variações no clima podem alterar a temperatura ambiental, o vento, a chuva, umidade e a seca que poderão afetar direta ou indiretamente a distribuição do patógeno, as taxas de reprodução do vetor e os meios de transmissão.

Os artigos (7) e (12) indicaram que as infecções levadas por vetores dependerão da variação espacial, sazonalidade e flutuações do clima, que podem alterar os nichos ecológicos dos vetores de doenças. O artigo (5) deu ênfase a forte dependência da malária e a dengue com as condições climáticas. O estudo (13) trouxe a relação da incidência ou prevalência de infecções com a localização dos países e situações socioeconômicas, poluição e perturbações na saúde.

\subsection{Mudanças climáticas e atividades humanas como determinantes significativas nas doenças infecciosas}

Para os autores da referência (1) as mudanças demográficas, comportamento humano como viagens, comércio, as mudanças ecológicas, tecnologia, indústria, a adaptação microbiana e o colapso das medidas de saúde pública já se configuram como fatores suficientes para o surgimento e disseminação de doenças infecciosas. Estas mudanças afetam a reprodução, desenvolvimento e sobrevivência de patógenos, hospedeiros na interação com humanos. Como mediadas para redução de danos, os autores apontam a vigilância global eficaz, vontade política global e colaboração multidisciplinar, que serão úteis na resposta a quaisquer ameaças futuras.

Nas pesquisas dos autores da referência (5) Vários fatores antropogênicos podem estar envolvidos no surgimento de novos vírus: através do desmatamento e mudança no manuseio da terra/solo. O consumo de carne de caça também é um driver para doenças infecciosas emergentes no Brasil. O comércio de animais selvagens e especialmente o comércio de animais de estimação tem sido fortemente implicados como um impulsionador do surgimento de doenças infecciosas. Segundo os autores, a vigilância sanitária aprimorada e bem direcionada fornecerá um melhor conhecimento para a investigação dos riscos específicos de contingência de doenças.

Na referência (9), os estudos revelam que o choque entre a destruição do ozônio, a perda da biodiversidade e degradação generalizada da terra em conjunto com a expansão agrícola, invasão de habitat, caça e comércio se configuram como colapso entre humanos e vida selvagem que podem ampliar os processos de transmissibilidade de doenças. Ressaltam que a saúde pública deve não apenas controlar e conter, mas também buscar prevenir o surgimento de doenças causadas pelo clima, olhando a montante para lugares, pessoas e práticas reais. Tal orientação revela a interdependência de conhecimentos específicos da disciplina e colaboração interdisciplinar, e a importância de colaborar com aliados, incluindo pesquisadores dentro e fora das ciências da saúde.

Em conclusão, a referência (13) aborda a exacerbação populacional urbano desordenado, o mecanismo de globalização e ampliação do intercâmbio internacional. Paralelamente, o clima em descontrolado aquecimento também se 
mostra como um importante fator na ação de transmissão de patógenos ao homem. A temperatura global inflada afeta os mosquitos vetores pois reduz o ciclo de desenvolvimento de larvas e aumenta de forma acelerada a população de vetores adultos.

\subsection{Doenças infecciosas e sua relação com as variações de temperatura}

Consoante com os pesquisadores da referência (2), conforme os hospedeiros se adaptam a temperaturas mais altas, os patógenos (vírus, bactérias ou fungos) devem seguir o exemplo para sobreviver e assim adquirirem maior capacidade de replicação em temperaturas exacerbadas. Se Não houver intervenção, a medicina precisará enfrentar novas doenças infecciosas para as quais não tem experiência.

Na referência (8) os estudiosos afirmam A periodicidade irregular do El Niño Oscilação Sul (ENOS) promove impactos na mudança dos indicadores de temperatura e precipitação no risco de doenças. A hipótese era que o ENOS estaria associado a mudanças na epidemiologia de patologias infecciosas na região oeste dos Estados Unidos mais conectada do que em outras regiões. Observaram que havia esse impacto do ENOS no risco de doenças propagadas por vetores na região oeste dos EUA, sem mudanças na carga de doenças em outras regiões. Este impacto foi concentrado em um intervalo de 10-12 meses e parecia ser amplo nas doenças transmitidas por carrapatos. Este achado tem relação com os efeitos potenciais da precipitação e temperaturas elevadas em vetores de carrapatos, bem como reservatórios de doenças de roedores aumentados embora tais observações não sejam universais.

\subsection{A influência do clima sobre os patógenos e hospedeiros/vetores}

Os pesquisadores das referências (3), (4) e (9) concordam que o embate das variáveis do clima, sobre os patógenos, podem ocorrer de forma direta ou indireta. No artigo (3) nas regiões norte (Ártico), inferem que vetores artrópodes (carrapatos, mosquitos e animais reservatórios) podem estender sua distribuição para o Norte como resultado de mudanças associadas ao aquecimento do clima. (4) afirma que a temperatura ambiental, os ventos e os padrões de precipitação afetam indiretamente a distribuição do patógeno, as taxas de reprodução do vetor e os meios de transmissão. O (9), cita a temperatura, a chuva, unidade e o vento em índices elevados influenciam na transmissão, reprodução e seletividade adaptativa de patógenos.

Os estudos de (7) e (12) trouxeram a relação da variação espacial, sazonalidade, variação plurianual e a combinação das escalas espaciais e temporais à influência na biologia do desenvolvimento de patógenos e vetores. As temperaturas exacerbadas encurtam a duração do ciclo de parasitas e aumento de chuvas facilita a formação de criadouros de insetos vetores. Afirmam que, embora as previsões de chuvas no próximo século sejam incertas, há evidências de que a aridez em muitas regiões aumentará muito nas próximas décadas. Os atrasos projetados no início sazonal das chuvas de monções, conforme o clima aquece são robustos, e derivam de uma termodinâmica atmosférica bem compreendida. Um trabalho interdisciplinar rigoroso, no qual os cientistas do clima trabalham em colaboração com os modeladores de doenças, tem, portanto, o potencial de levar à descoberta de restrições emergentes na futura incidência de comorbidades.

No artigo (5), o clima pode favorecer a criação de ambientes propícios à formação de criadouros de vetores de doenças e também gerar doenças e agravos diretos em virtude de fenômenos climáticos, como inundações. Embora haja evidências da interferência do clima no acontecimento de distintas doenças, ainda não se conhecem todas as doenças sensíveis ao clima. Além disso, é necessário compreender os processos de exposição, ou seja, quais variáveis climáticas podem influenciar a ocorrência de doenças e como esse processo se dá. Há a necessidade de ampliar os países estudados, considerando os impactos previstos pelas mudanças no clima, da mesma forma como a diversidade ambiental, climática e socioeconômica incidem de maneiras diversas as patologias sensíveis ao clima. 
Conforme o artigo (13), embora os impactos destas mudanças sobre as doenças sejam detectados em todo mundo, as formas e os graus desses impactos variam muito. Por causa do complexo processo de interações entre as variáveis climáticas e os componentes dos danos infecciosos (patógeno, hospedeiro e ambiente de transmissão) para as comorbidades específicas os impactos são distintos em distintas escalas geográficas, para diferentes doenças e, as vezes controversos. Além do que foi abordado, o acontecimento de comorbidades infecciosas está ligada a muitos outros fatores além do clima, cintando como exemplo, os antropogênicos.

\section{Conclusão}

O quantitativo de pesquisas brasileiras acerca da temática foi inferior ao esperado, que talvez se justifique pela escolha das palavras-chave. Os estudos que subsidiaram esta pesquisa, em sua totalidade, concordaram que existem relações entre as variáveis climáticas e as doenças infecciosas, de formas variadas. Ainda apontou como as atividades antropogênicas interferem, de forma definitiva, nas mudanças do clima.

Observa-se dois grandes desafios em pauta: o fenômeno da globalização, que eleva a economia, diminui fronteiras e abre portas a novas tecnologias; na mesma direção, a agricultura como fator que acarreta grandes choques ao meio ambiente mas que é de extrema necessidade para a sobrevivência humana. Tudo isso gera uma emergência em encontrar respostas às variações ou variedades de climas globais na busca de um desenvolvimento sustentável o que torna um grande desafio para uma a sociedade ainda em ascensão.

Apesar deste estudo trazer apenas um estudo que aborda sobre a atual pandemia vivenciada (Covid-19), é notório que há estreita relação desta com a interação homem-clima e homem-animal. Com dito anteriormente, a temperatura, a umidade, a intervenção antropogênica entre outros, alteram ou influenciam de alguma forma na disseminação desta infecção, isto adverte para a importância de uma aproximação inter e multidisciplinar, mas sobre uma perspectiva ética sustentável e justa que permita a convivência de todas as pessoas em dignidade e com todos os seres vivos em equilíbrio.

As questões debatidas acima fornecem subsídios para novas pesquisas e sinalizam para a questão da reduzida produção científica brasileira acerca dos temas sobre o clima, visto que o Brasil tem uma grande diversidade climática. Destaca-se a relevância de aumentar os contingentes de pesquisas voltadas a este tema, objetivando fornecer bases para uma previsão das consequências futuras das mudanças climáticas para a saúde da humanidade e a adoção de medidas de adaptação eficientes.

\section{Referências}

Abreu, A. M., et al. (2020). A interface entre saúde, mudanças climáticas e uso do solo no Brasil: uma análise da evolução da produção científica internacional entre 1990 e 2019. Saúde soc, 29 (2), e 180866.

Bardin, L. (2016). Análise de Conteúdo.

Barros, D. F., et al. (2021). Liderança do Enfermeiro na Atenção Primária à Saúde: Uma revisão integrativa. Research, Society and Development. 10 (1), 1-9, e26110110099.

Casadevall, A., et al. (2020). Climate change brings the specter of new infectious diseases. Journal Of Clinical Investigation, 130 (2), $553-555$.

Confalonieri, U. E. C., Menezes, J. A. \& Souza, C. M. de. (2015). Climate change and adaptation of the health sector: the case of infectious diseases. Virulence, $6(6), 554-557$.

Duarte, J. L. et al., (2019). Variabilidade climática e interações por doenças diarreicas infecciosas em um município da Amazônia Ocidental Brasileira. Ciênc. Saúde Coletiva. 24 (8), 2959-2970.

Fisman, D. N., Tuite, A. R. \& Brown, K. A., (2016). Impact of El Niño Southern Oscillation on infectious disease hospitalization risk in the United States. Procedings Of The National Academy Of Sciences, 113 (51), 14589-14594.

Gislason, M. K., et al. (2015). Climate change, health and infectious disease. Virulence. 6 (6), 539-542. 
Research, Society and Development, v. 10, n. 2, e7910212126, 2021

(CC BY 4.0) | ISSN 2525-3409 | DOI: http://dx.doi.org/10.33448/rsd-v10i2.12126

Khan, M. D., et al. (2019). Aggravation of Human Diseases and Climate Change Nexus. International Journal Of Environmental Research And Public Health. $16(15), 1-26$

Lima-Camara, T. N. (2016). Emerging arboviruses and public health challenges in Brazil. Revista de Saúde Pública. 50, 1-7.

Lima, T. F. S., et al. (2020). Fatores que afetam a saúde mental de estudantes universitários indígenas: revisão integrativa da literatura. In: Jorge, W. J. (Ed) Ciências da Saúde - Teoria e Prática (pp. 563-576). Uniedusul.

Lobão, L. M. \& Rodrigues, B. S. S. L. (2019). Mudanças ambientais de origem antrópica e sua relação com o adoecimento humano. Revista Saúde Dinâmica. $1(1), 33-51$.

Metcalf, C. J. E., et al. (2017). Identifying climate drivers of infectious disease dynamics: recent advances and challenges ahead. Proceedings Of The Royal Society B: Biological Sciences. 284 (1860), 1-10.

Moher, D., et al. (2009). Preferred reporting items for systematic reviews and meta-analyses: The PRISMA statement. PLoS Medicine. 6 (7).

Nava, A., et al. (2017). The Impact of Global Environmental Changes on Infectious Disease Emergence with a Focus on Risks for Brazil. Ilar Journal. 58 (3), 393-400.

Omazic, A., et al. (2019). Identifying climate-sensitive infectious diseases in animals and humans in Northern regions. Acta Veterinaria Scandinavica, 61 (1), $1-12$.

Pereira, A. S., et al. (2018). Metodologia da pesquisa científica. Ed. UAB/NTE/UFSM.

Sabin, N. S., et al. (2020). Implications of human activities for (re)emerging infectious diseases, including COVID-19. Journal Of Physiological Anthropology. 39 (1), 1-12.

Sousa, T. C. M., et al. (2018). Doenças sensíveis ao clima no Brasil e no mundo: revisão sistemática. Revista Panamericana de Salud Pública. 42, 1-10, 2018.

Teixeira, E. (2013). Revisão Integrativa da Literatura passo-a-passo \& covergências com outros métodos de revisão, REUFPI.

Ursi, E. S. \& Galvão, C. M. (2015). Avaliação das escalas de risco para úlcera por pressão em pacientes críticos: uma coorte prospectiva. Acta Paul Enferm. $23(1), 28-35$.

Wang, Y., et al. (2015). A Method for Screening Climate Change-Sensitive Infectious Diseases. International Journal Of Environmental Research And Public Health. 12 (1), 767-783.

Wu, X. et al. (2016). Impact of climate change on human infectious diseases: empirical evidence and human adaptation. Environment International. 86, 14-23. 